Article

\title{
Dynamics Analysis of Misalignment and Stator Short-Circuit Coupling Fault in Electric Vehicle Range Extender
}

\author{
Xiaowei Xu ${ }^{1, *}$, Jingyi Feng ${ }^{1}$, Hongxia Wang ${ }^{1}$, Nan Zhang ${ }^{2}$ and Xiaoqing Wang ${ }^{3}$ \\ 1 School of Automobile and Traffic Engineering, Wuhan University of Science and Technology, Wuhan 430081, \\ China; fengjingyi97@gmail.com (J.F.); whx631@163.com (H.W.) \\ 2 School of Computer Science, University of Nottingham, Nottingham NG7 2RD, UK; \\ psxnz1@nottingham.ac.uk \\ 3 Department of Applied Engineering, Jacksonville State University, Jacksonville, AL 36265, USA; \\ xq7.wang@gmail.com \\ * Correspondence: xuxiaowei@wust.edu.cn; Tel.: +86-027-6889-6947
}

Received: 23 July 2020; Accepted: 17 August 2020; Published: 25 August 2020

\begin{abstract}
Due to the complex structure and wide excitation of the range extender, the misalignment and stator short-circuit coupling fault can easily occur. Therefore, it is necessary to study the coupling fault mechanism of the range extender, analyze the cause of the fault and the fault evolution law, and research the coupling fault characteristics. To reveal the mechanism of misalignment and stator-short-circuit coupling fault, the misalignment mechanism was analyzed and the bending and torsion electromagnetic stiffness of the generator in the stator short-circuit fault was derived. Then the dynamic model of bending and torsion coupling for the generator was established. Furthermore, we used the Runge-Kutta method to study the vibration response characteristics of generator rotor under coupling fault. Then through finite element analysis, the feasibility of coupled fault diagnosis was verified. The results show that the response of the generator rotor not only has the frequency component of single faults, but also new frequency components such as 4.0 and 6.0 harmonic amplitudes of radial vibration and 3.0 harmonic amplitudes of torsional vibration, respectively.
\end{abstract}

Keywords: electric vehicle range extender; misalignment and stator short-circuit coupling fault; failure mechanism analysis; dynamic analysis; numerical analysis

\section{Introduction}

As a smooth transition model from the hybrid electric vehicle to a pure electric vehicle, the Range Extended Electric Vehicle (REEV) is of significant interest because of the high efficiency, small battery capacity and long driving mileage. It is an important way to solve the energy crisis, environmental pollution and other problems [1]. The range extender is the auxiliary power unit of the REEV, which is composed of a coaxially connected engine and generator. However, it does not start when driving a short distance and is not easily found in the case of failure, which can cause sudden accidents and affect the stability and safety of the whole vehicle $[2,3]$. The range extender has a complex structure and many sources of excitation. According to statistics, more than $60 \%$ of faults in rotating machinery systems are related to rotor misalignment [4]. Misalignment of the motor shafting will cause abnormal vibrations of the unit, and the vibration will also accelerate the abrasion of the winding insulation layer and induce stator short-circuit faults $[5,6]$. The stator winding short-circuit is a common electrical fault, accounting for approximately $30 \%$ of all generator failures [7]. The circulating current caused by the stator short-circuit will cause the winding coils to partly heat up and accelerate the insulation layer aging, which will cause phase-to-phase short-circuit faults that further enhance the unit vibration [8-10]. 
The vibration also will cause the shafting to deflect and tilt, finally leading to misalignment faults [11]. It can be seen that misalignment faults and stator short-circuit faults are mutually affected. The study of the vibration characteristics of the system under misalignment and stator short-circuit coupling faults can provide a theoretical basis for unit condition monitoring and fault diagnosis.

It is difficult to simulate all fault types and fault degrees, which may permanently damage the motor or test bench, resulting in high test costs, as well as affecting the safety of laboratory personnel and equipment. Therefore, finite element analysis method is often used to study the coupling fault. However, the solution obtained by finite element analysis is approximate, the accuracy of the solution is relatively large, and the accuracy of the solution depends on the level of modeling and boundary conditions, whether the simulation of load cases is true, etc. By establishing a mathematical model to study the dynamic characteristics, the coupling fault can be analyzed more accurately in theory [12-14].

Many scholars have studied the misalignment fault and stator short-circuit fault by numerical analysis. At present, the inter-turn short-circuit of the stator winding and misalignment faults have been thoroughly studied locally and abroad. Liao has analyzed the dynamic load generated by misalignment of the permanent magnet synchronous motor and revealed the characteristics of generator vibration caused by misalignment [15]. It shows that the misalignment fault is one of the most important causes of generator failure. Li has studied the nonlinear dynamic behavior of the generator rotor system with parallel misalignment faults under the condition of rotor asymmetry [16]. Wang analyzed the vibration mechanism and movement law of rotor misalignment, and obtained the typical characteristics of misalignment faults [17]. The study of inter-turn short-circuits in generator stator winding is mostly based on the change of electrical and mechanical parameters, such as current, vibration frequency of stator and rotor, etc. Wu analyzed the vibration characteristics of the generator caused by the inter-turn short-circuit using the magnetic density method and the energy method [18]. He used a combination of analytical analysis, numerical calculation, and experimental verification to analyze the stator vibration characteristics under different positions of the stator short-circuit fault [19]. Ma theoretically analyzed the generator vibration characteristics caused by stator inter-turn short-circuits using the magnetic density method and energy method [20]. However, most of the above studies are based on a single misalignment fault or stator short-circuit fault, seldom considering the coupling effect and fault mechanism analysis [21,22].

Based on the analysis of the misalignment fault excitation mechanism and derivation of the generator electromagnetic stiffness under a stator short-circuit, a dynamic model of the rotor system of the generator of range extender is established in this paper. By using numerical methods, this paper has studied the law and characteristics of rotor vibration response during the coupling fault of misalignment and stator short-circuit. Thus, it provides a theoretical basis for motor fault diagnosis and prevention.

\section{Dynamic Modeling of Range Extender}

\subsection{Dynamic Modeling}

Because misalignment faults and stator short-circuit faults involve many factors, in order to highlight the main problem, the range extender is simplified to the dynamic model shown in Figure 1. Ignoring the mass of the shaft, the rotor of the generator is simplified into a wheel disk with a mass of $m_{2}$. The coordinate of the rotation center $\mathrm{O}_{2}$ is $\left(m_{2}\right)$. The mass of the journal is $m_{1}$, and the displacement of it is $\left(x_{1}, y_{1}\right)$. The system rotation speed is $\omega$. The rotor is affected by misalignment excitation of coupling and electromagnetic excitation of the generator during operation.

Both ends of the generator are supported by guide bearings. The oil film force can be expressed by the linear combination of journal displacement and journal speed. The system of differential equations of motion considering misalignment and inter-turn short-circuit faults is: 


$$
\left\{\begin{array}{l}
m_{1} \ddot{x}_{1}+c_{1} \dot{x}_{1}+0.5 k_{w}\left(x_{1}-x_{2}\right)=f_{x} \\
m_{1} \ddot{y}_{1}+c_{1} \dot{y}_{1}+0.5 k_{w}\left(y_{1}-y_{2}\right)=f_{y} \\
m_{2} \ddot{x}_{2}+c_{2} \dot{x}_{2}+k_{w}\left(x_{2}-x_{1}\right)+K_{e x x} x_{2}+K_{e x y} y_{2}=m_{2} e_{0}\left(\dot{\phi}^{2} \cos \phi+\ddot{\phi} \sin \phi\right)+c_{2} e_{0} \dot{\phi} \sin \phi-f_{e x}+F_{c x} \\
m_{2} \ddot{y}_{2}+c_{2} \dot{y}_{2}+k_{w}\left(y_{2}-y_{1}\right)+K_{e y y} y_{2}+K_{e y x} x_{2}=m_{2} e_{0}\left(\dot{\phi}^{2} \sin \phi-\ddot{\phi} \cos \phi\right)-c_{2} e_{0} \dot{\phi} \cos \phi-f_{e y}+F_{c y} \\
I_{p} \ddot{\varphi}+c_{t} \dot{\varphi}+k_{t} \varphi+K_{t e} \varphi=e_{0}\left(k_{w}\left(y_{2}-y_{1}\right) \cos \phi-k_{w}\left(x_{2}-x_{1}\right) \sin \phi\right)-m_{e}
\end{array}\right.
$$

among them

$$
\left\{\begin{array}{l}
f_{x}=k_{x x} x_{1}+k_{x y} y_{1}+c_{x x} \dot{x}_{1}+c_{x y} \dot{y}_{1} \\
f_{y}=k_{y y} y_{1}+k_{y x} x_{1}+c_{y y} \dot{y}_{1}+c_{y x} \dot{x}_{1}
\end{array}\right.
$$

To facilitate the calculation, a dimensionless quantity change is introduced:

$$
X_{i}=x_{i} / d, Y_{i}=y_{i} / d,(i=1,2), \tau=\omega t
$$

where $d$ is the unit length, $x_{i}, y_{i}$ are dimensionless displacement, and $\tau$ is dimensionless time.

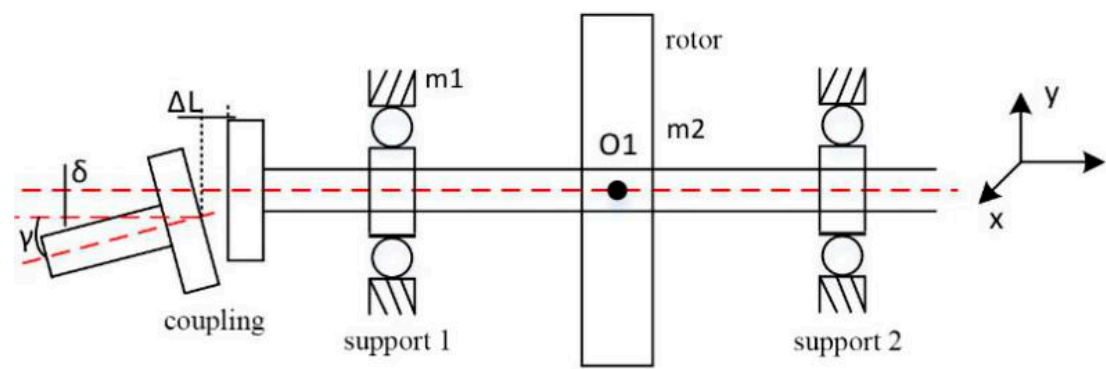

Figure 1. Dynamic model of range extender.

\subsection{Excitation Analysis of Misalignment Fault}

Among the three common faults of parallel misalignment, angular misalignment, and comprehensive misalignment, the last fault is most likely to occur. Figure 2 is a schematic diagram of coupling misalignment. $O_{1}$ and $O_{2}$ are the rotation centers of the left and right half of the coupling. $O$ and $k$ are respectively the stationary and dynamic centers of the coupling housing, $\theta$ is the angle that the half coupling has rotated. The dynamic center performs a circular motion under failure condition. When the two half couplings rotate at angular velocity $d \theta / d t=\omega$, the expression of the coupling housing dynamic center $k$ can be obtained according to the deformation geometric relationship as [23]:

$$
\left\{\begin{array}{l}
x=\Delta E \cos \theta \cos \theta-0.5 \Delta E=0.5 \Delta E \cos 2 \theta \\
y=\Delta E \sin \theta \cos \theta=0.5 \Delta E \sin 2 \theta
\end{array}\right.
$$

where: $\Delta E=\Delta \delta+\Delta L \tan \gamma$ is the comprehensive misalignment amount of coupling, when parallel misalignment happens, $\Delta E=\Delta \delta$. When the angle misalignment occurs, $\Delta E=\Delta L \tan \gamma . \Delta L$ is the installation distance between the two half couplings. $\Delta \delta$ is the amount of parallel misalignment between the two halves of the coupling. $\gamma$ is the amount of angular misalignment between them. Thus, the dynamic center line velocity and angular velocity can be expressed as follows:

$$
\begin{aligned}
& v_{k}=\sqrt{(d x / d t)^{2}+(d y / d t)^{2}}=\omega \Delta E \\
& \omega_{k}=\frac{v_{k}}{\Delta E / 2}=2 \omega
\end{aligned}
$$

It can be seen that the coupling housing rotates at twice the rotation speed of the system, and when the system rotates by $\theta$ angle, $k$ points will rotate through the $2 \theta$ angle. Its rotation center is at the middle point of the axis connecting line of the two half couplings, so the characteristic frequency of misalignment fault is twice that of the angular frequency of the rotating shaft. Due to the relatively 
large mass of the coupling housing $m_{0}$, this rotary motion is equivalent to an unbalanced mass acting on the system. So, the inertial force of the dynamic center of the housing is relative to the static center under the misalignment state, that is, the misalignment fault excitation. It can be expressed as follows:

$$
\left\{\begin{array}{l}
F_{c x}=-m_{0} \ddot{x}=-m_{0} \Delta E\left[2 \dot{\theta}^{2} \cos 2 \theta+\ddot{\theta} \sin 2 \theta\right] \\
F_{c y}=-m_{0} \ddot{y}=-m_{0} \Delta E\left[2 \dot{\theta}^{2} \sin 2 \theta-\ddot{\theta} \cos 2 \theta\right]
\end{array}\right.
$$

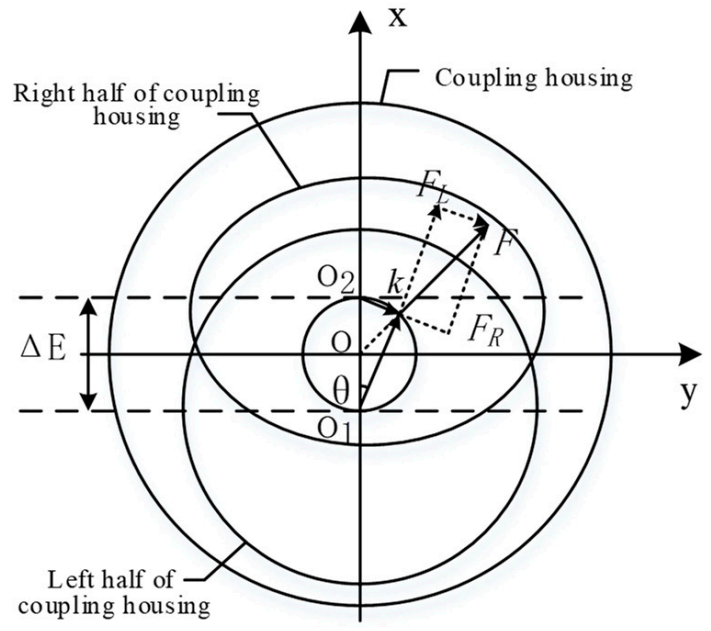

Figure 2. Schematic of relative movement for the coupling halves and housing.

\subsection{Excitation Analysis of Stator Short-Circuit}

After the generator of the range extender has encountered the stator short-circuit fault, the electromagnetic force on the rotor will change. This section uses the energy method to calculate the unbalanced electromagnetic tension and torque received by the generator during the short-circuit, and further derives the bending electromagnetic stiffness and torsional electromagnetic stiffness. The unbalanced electromagnetic tension and electromagnetic torque of a generator are variable loads, the size and direction of which vary with time, and are related to the rotor bending and torsional vibration response as well as rotor movement position. If the electromagnetic force and the electromagnetic torque are considered as the stiffness form, it can, not only avoid a series of complicated calculations related to the vibration response of the rotor, but also solve the problem of the direction changing with the movement of the rotor, which is a simple and convenient processing function.

\subsubsection{Air Gap Permeance Calculation}

Due to factors such as manufacturing, assembly, and operation, the generator has different degrees of air gap eccentricity. After the short-circuit fault occurs in the stator, its actually equivalent to a compound fault of air gap eccentricity and stator short-circuit. The air gap eccentricity affects the electromagnetic force and torque by affecting the air gap permeance and the air gap magnetic field has asymmetric distribution under the air gap eccentricity. Figure 3 shows the rotor air gap eccentricity, where the point $O_{1}$ is the geometric center of the outer circle of the rotor, of which the origin of the coordinates is $(x, y) . \delta$ is the size of the air gap; the eccentricity of the air gap $e=\sqrt{x^{2}+y^{2}}$, in which $x, y$ are respectively the components in $x$-axis and $y$-axis of eccentricity; $\alpha$ is the angle between the circumference and $x$-axis when the size of the air gap $=\delta, \beta$ is the angle between minimum air gap position and $y$-axis. 


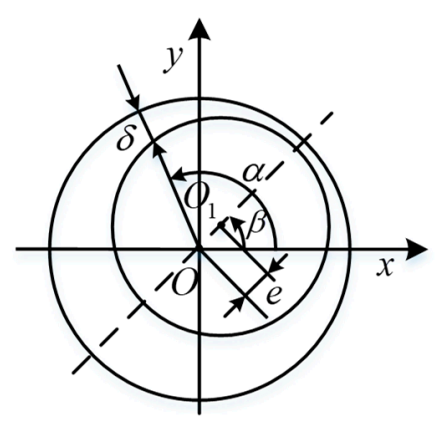

Figure 3. Schematic diagram of air gap eccentricity.

The radial air gap length from the stator and rotor is:

$$
\delta(\alpha, \mathrm{t})=\delta_{0}-e \cos (\alpha-\beta)
$$

In the formula: $\delta_{0}$ is the uniform air gap size. The expression of air gap permeability per unit area under eccentricity is:

$$
\Lambda(\alpha, \mathrm{t})=\frac{\mu_{0}}{\delta(\alpha, \mathrm{t})}=\frac{\mu_{0}}{k_{\mu} \delta_{0}[1-\varepsilon \cos (\alpha-\beta)]}=\Lambda_{0} \sum_{n=0}^{\infty} \varepsilon^{n} \cos (\alpha-\beta)^{n}
$$

where: $k_{\mu}$ is the saturation, $\varepsilon=\mathrm{e} / k_{\mu} \delta_{0}$ is the effective relative eccentricity, $\Lambda_{0}$ is the uniform air gap permeability of the generator, and $n$ is the Taylor series expansion order.

By means of De Moivre's formula, the air gap permeability is converted into a sum after a series of calculations:

$$
\Lambda(\alpha, \mathrm{t})=\Lambda_{0} \sum_{n=0}^{\infty}\left(\frac{\varepsilon}{2}\right)^{n}\left[\cos n(\alpha-\beta)+C_{n}^{1} \cos (\mathrm{n}-2)(\alpha-\beta)+\ldots+C_{n}^{n} \cos (\mathrm{n}-2 n)(\alpha-\beta)\right]
$$

It can be derived from Equation (9) that among the components of the air gap permeance, $n=0$ is the largest weight, and the components of $n \neq 0$ are caused by the air gap eccentricity. Since $\varepsilon$ is far less than 1, the weight caused by the air gap eccentricity is smaller with the value increase of $n$. So just taking the first three terms of the air gap permeance can meet the calculation accuracy requirements.

The air gap permeance per unit area is:

$$
\Lambda(\alpha, \mathrm{t})=\Lambda_{0}\left[\left(1+\frac{\varepsilon^{2}}{2}\right)+\varepsilon \cos (\alpha-\beta)+\frac{\varepsilon^{2}}{2} \cdot \cos 2(\alpha-\beta)\right]=\Lambda_{0}\left[1+\frac{x^{2}+y^{2}}{2 \sigma^{2}}+\frac{x}{\sigma} \cos \alpha+\frac{y}{\sigma} \sin \alpha+\frac{x^{2}-y^{2}}{2 \sigma^{2}} \cos 2 \alpha+\frac{x y}{\sigma^{2}} \sin 2 \alpha\right]
$$

where: $x=\sigma \varepsilon \cos \beta, y=\sigma \varepsilon \sin \beta, \sigma=k_{u} \delta_{0}$.

\subsubsection{Air Gap Magnetic Field Calculation}

During normal operation, the combined air gap magnetic potential of the stator and rotor rotates synchronously with the rotor. The combined magnetic potential is:

$$
f(\alpha, t)=F_{s} \cos (\omega t-p \alpha)+F_{r} \cos (\omega t-p \alpha+\theta+\varphi+\pi / 2)
$$

In the formula: $p$ is the number of pairs of magnetic poles, and $\omega$ is the frequency of the rotor rotation angle, $F_{S}$ and $F_{r}$ are the amplitudes of the fundamental wave potentials of the generator and the rotor windings, respectively. The phases of the fundamental wave of stator and rotor are different, and the fundamental wave phase of the rotor is ahead of the stator $\theta+\varphi+\pi / 2$ degrees, where $\theta$ is the inner power angle of the generator and $\varphi$ is the rotor torsional vibration angle. A schematic diagram of stator windings inter-turn short-circuit is shown in Figure 4. 


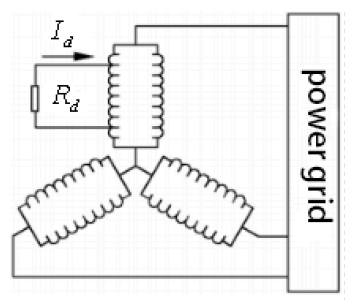

Figure 4. Stator windings short-circuit schematic.

When a short-circuit fault between the stator windings of the generator occurs, a short-circuit current will be generated. The current will cause a pulsating magnetic field that fluctuates around the short-circuit axis. The pulse frequency is the rated electrical frequency of the generator. The magnetic potential caused by the short-circuit is:

$$
f(\alpha, \mathrm{t})=F_{d} \cos (\omega \mathrm{t}) \cos (p \alpha)
$$

It can be decomposed into two rotating magnetic potentials:

$$
f(\alpha, \mathrm{t})=F_{d+} \cos (\omega \mathrm{t}-p \alpha)+F_{d-} \cos (\omega \mathrm{t}+p \alpha)
$$

It can be seen from Formula (13) that the pulsating magnetic potential consists of two parts: the magnetic potential rotating in the same direction with the rotor and the magnetic potential rotating in the opposite direction with the rotor. The first part does not generate additional induced potential in the rotor winding, and the second part will induce an additional harmonic wave potential in the rotor winding at a frequency twice that of the electrical frequency. So, the excitation current of the rotor is:

$$
I_{f}(\mathbf{t})=I_{f 0}+I_{f 2} \cos 2 \omega t
$$

The main magnetic density distribution of the air gap generated by the excitation current in the rotor winding is:

$$
B(t)=B_{0}+B_{2} \cos 2 \omega t
$$

The effective value of the induced electromotive force of the three-phase stator winding under this main magnetic density is:

$$
E(\mathrm{t})=2 q f w k_{w} B l \tau=K B
$$

where: $q$ is the number of slots per pole and phase of the stator; $f$ is the electrical frequency, $\omega$ is the number of single-phase series turns of the stator winding, $K_{\omega}$ is the fundamental winding coefficient, $l$ is the conductor length, $\tau$ is the pole distance, and the coefficient $K=2 q f w k_{w} l \tau$.

The three-phase winding induced potential is:

$$
\left\{\begin{array}{l}
e_{a}(\mathrm{t})=E \cos \omega \mathrm{t}=K B_{0} \cos \omega \mathrm{t}+\left(K B_{2} / 2\right) \cdot \cos \omega \mathrm{t}+\left(K B_{2} / 2\right) \cos 3 \omega \mathrm{t} \\
e_{b}(\mathrm{t})=E \cos (\omega \mathrm{t}-\pi / 3)=K B_{0} \cos (\omega \mathrm{t}-\pi / 3)+\left(K B_{2} / 2\right) \cos (\omega \mathrm{t}+\pi / 3)+\left(K B_{2} / 2\right) \cos (3 \omega \mathrm{t}-\pi / 3) \\
e_{c}(\mathrm{t})=E \cos (\omega \mathrm{t}+\pi / 3)=K B_{0} \cos (\omega \mathrm{t}+\pi / 3)+\left(K B_{2} / 2\right) \cos (\omega \mathrm{t}-\pi / 3)+\left(K B_{2} / 2\right) \cos (3 \omega \mathrm{t}+\pi / 3)
\end{array}\right.
$$

From Equation (17), it can be determined that the three-phase winding induced potentials are composed of three sub terms. The first term will form the armature reaction magnetic potential that rotates forward at the fundamental frequency speed, and the second term will form the potential that rotates inverse at the fundamental frequency speed; the third term will form the armature reaction magnetic potential that rotates forward at three times the frequency of the fundamental speed. Therefore, the combined magnetic potential of the air gap under the stator winding inter-turn short-circuit fault can be approximately expressed as: 


$$
\begin{aligned}
f(\alpha, \mathrm{t})= & F_{s 1+} \cos (\omega \mathrm{t}-p \alpha)+F_{s 1-} \cos (\omega \mathrm{t}+p \alpha)+F_{s 3+} \cos (3 \omega \mathrm{t}-p \alpha)+F_{r 1+} \cos (\omega \mathrm{t}-p \alpha+\psi+\pi / 2)+ \\
& F_{r 1-} \cos (\omega \mathrm{t}+p \alpha-\psi-\pi / 2)+F_{r 3+} \cos (3 \omega \mathrm{t}-p \alpha+\psi+\pi / 2)
\end{aligned}
$$

where $F_{s 1+}, F_{s 1-}, F_{s 3+}$ are the amplitude of the armature reaction magnetic potential, and $F_{s 1-}=$ $F_{s 3+}, F_{r 1+}, F_{r 1-}, F_{r 3+}$ are the amplitude of the magnetic potential generated by the excitation current, $F_{r 1-}=F_{r 3+} \psi=\varphi+\theta$.

Introducing $v$ as the characterization parameter of the stator inter-turn short-circuit degree [24], the relationship between the amplitude of the magnetic potentials is:

$$
v=\frac{F_{s 3+}}{F_{s 1+}}=\frac{F_{r 3+}}{F_{r 1+}}
$$

It can be determined from Equation (19) that, with the increase of the value, the amplitudes of the reverse rotating magnetic potential and the triple-frequency rotating magnetic potential caused by the short-circuit in the air gap synthesized magnetic potential will increase.

\subsubsection{Electromagnetic Stiffness Calculation in Case of Short-Circuit}

According to the electrical engineering that the air gap magnetic field energy of a generator is:

$$
W=\frac{R_{g} L^{\prime}}{2} \int_{0}^{2 \pi} \Lambda(\alpha, \mathrm{t}) \cdot f(\alpha, \mathrm{t})^{2} d \alpha
$$

where, $R_{g}$ is the inner radius of the stator and $L^{\prime}$ is the effective length of the rotor.

Bringing Equations (10) and (18) into the calculation of the air gap magnetic field energy, and taking the partial derivative of the vibration displacement $x, y$ of the rotor to obtain the unbalanced electromagnetic tensile force acting on the $\mathrm{x}$ and $\mathrm{y}$ direction, as well as then taking the derivative of rotor torsional vibration angle, gives the electromagnetic torque:

$$
\left\{\begin{array}{c}
F_{e x}=\frac{\partial W}{\partial x}=\frac{R_{g} L^{\prime} \Lambda_{0}}{2 \sigma^{2}} \cdot \int_{0}^{2 \pi}[(1+\cos 2 \alpha) x+\sigma \cos \alpha+y \sin 2 \alpha] \cdot f^{2}(\alpha, \mathrm{t}) d \alpha \\
F_{e y}=\frac{\partial W}{\partial y}=\frac{R_{g} L^{\prime} \Lambda_{0}}{2 \sigma^{2}} \cdot \int_{0}^{2 \pi}[(1-\cos 2 \alpha) y+\sigma \sin \alpha+x \sin 2 \alpha] \cdot f^{2}(\alpha, \mathrm{t}) d \alpha \\
M_{\mathcal{e}}=\frac{\partial W}{\partial(\varphi)}=\frac{R_{g} L \Lambda_{0}^{\prime}}{2} \int_{0}^{2 \pi}\left(1+\frac{x^{2}+y^{2}}{2 \sigma^{2}}\right) \frac{\partial f(\alpha, \mathrm{t})^{2}}{\partial \varphi} d \alpha
\end{array}\right.
$$

For electromagnetic torque, since $\varepsilon$ is much less than 1 , the air gap permeability can take the previous term. $\sin \varphi \approx \varphi-(1 / 6) \varphi^{3}, \cos \varphi \approx 1-(1 / 2) \varphi^{2}$. The trigonometric function containing the torsional vibration angle can be expanded and the higher terms can be omitted. Finally, taking the first term coefficient of the electromagnetic pull and electromagnetic torque, the bending and torsional electromagnetic stiffness of the generator can be obtained. The expressions are:

$$
\left\{\begin{array}{l}
F_{e x}=K_{e x x} \cdot x+K_{e x y} \cdot y+f_{e x} \\
F_{e y}=K_{e y x} \cdot x+K_{e y y} \cdot y+f_{e y} \\
M_{e}=K_{t e} \cdot \varphi+m_{e}
\end{array}\right.
$$

where, $K_{e x x}, K_{e x y}=K_{e y x}, K_{\text {eyy }}$ are the bending electromagnetic stiffness, $f_{\text {ex }}, f_{\text {ey }}$ are the electromagnetic load vector in the bending direction, $K_{t e}$ is the torsional electromagnetic stiffness, and $m_{e}$ is the electromagnetic load vector in the torsional direction. Among them: 


$$
\left\{\begin{array}{l}
K_{e x x}=\frac{R_{g} L^{\prime} \Lambda_{0}}{2 \sigma^{2}} \int_{0}^{2 \pi}(1+\cos 2 \alpha) \cdot f(\alpha, \mathrm{t})^{2} d \alpha \\
K_{e x y}=K_{e y x}=\frac{R_{g} L^{\prime} \Lambda_{0}}{2 \sigma^{2}} \int_{0}^{2 \pi}(\sin 2 \alpha) \cdot f(\alpha, \mathrm{t})^{2} d \alpha \\
K_{\text {eyy }}=\frac{R_{g} L^{\prime} \Lambda_{0}}{2 \sigma^{2}} \int_{0}^{2 \pi}(1-\cos 2 \alpha) \cdot f(\alpha, \mathrm{t})^{2} d \alpha \\
\left\{\begin{array}{l}
f_{e x}=\frac{R_{g} L^{\prime} \Lambda_{0}}{2 \sigma} \int_{0}^{2 \pi}(\cos \alpha) \cdot f(\alpha, \mathrm{t})^{2} d \alpha \\
f_{e y}=\frac{R_{g} L^{\prime} \Lambda_{0}}{2 \sigma} \int_{0}^{2 \pi}(\sin \alpha) \cdot f(\alpha, \mathrm{t})^{2} d \alpha
\end{array}\right.
\end{array}\right.
$$

\section{Numerical Analysis}

Runge Kutta with four or five orders and variable step size is used to integrate the differential equations of the range extender. The integration step size is $\mathrm{T} / 360$. Relevant parameters of the system are as follows: coupling mass $m_{0}=6 \mathrm{~kg}$, journal mass $m_{1}=2 \mathrm{~kg}$, rotor mass $m_{2}=25 \mathrm{~kg}$, rotor diameter inertia $I_{p}=0.131 \mathrm{~kg} \cdot \mathrm{m}^{2}$, rotor radius $R_{g}=0.24 \mathrm{~m}$, rotor length $L^{\prime}=0.3 \mathrm{~m}$, bending stiffness $k_{w}=5.2 \times 10^{5} \mathrm{~N} / \mathrm{m}$, torsional stiffness $k_{t}=2.53 \times 10^{6} \mathrm{~N} \times \mathrm{m} / \mathrm{rad}$, torsional damping ratio is 0.05, structural damping at the journal $c_{1}=10 \mathrm{~N} \cdot \mathrm{s} / \mathrm{m}$, structural damping $c_{2}=30 \mathrm{~N} \cdot \mathrm{s} / \mathrm{m}$, guide bearing stiffness and damping $k_{x x}=4.88 \times 10^{6} \mathrm{~N} / \mathrm{m}, c_{x x}=3.1 \times 10^{4} \mathrm{~N} \cdot \mathrm{s} / \mathrm{m}$, eccentricity $e_{0}=0.1 \mathrm{~mm}$, uniform air gap size $\delta_{0}=1 \mathrm{~mm}$, air permeability coefficient $\mu_{0}=4 \pi \times 10^{-7} \mathrm{H} / \mathrm{m}$, pole pair number is 1 , three-phase fundamental wave magnetic potential amplitude of generator rotor and stator winding $F_{r}=135.6 \mathrm{At}, F_{s}=1520 \mathrm{At}$, and generator speed is $1500 \mathrm{r} / \mathrm{min}$.

\subsection{Normal Condition}

Figure 5 shows the frequency spectrum of horizontal and torsional vibration of the generator rotor during normal operation. When the motor is working under normal conditions, the horizontal vibration is dominated by 1 harmonic frequency, and the torsional vibration is dominated by the zero frequency component. The combined magnetic potential of the stator and rotor rotates synchronously at the basic frequency. No negative sequence component and high frequency component are generated. The radial electromagnetic force is mainly the fundamental frequency component. It is affected by the direct current component of the magnetomotive force on the rotor that the electromagnetic torque is constant. Therefore, the frequency of the generator rotor radial vibration is mainly 1 harmonic of the fundamental frequency under electromagnetic excitation. Because the electromagnetic torque generated by the DC component does not have vibration properties, it will not excite the rotor to generate torsional vibration. The main component of it is zero harmonic frequency.

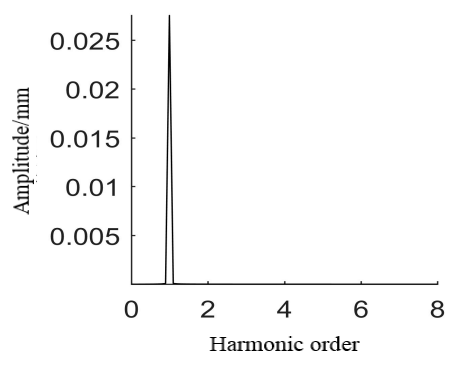

(a)

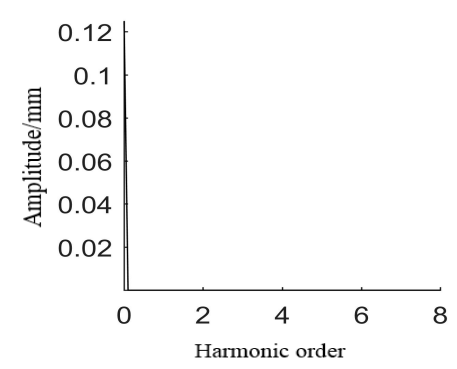

(b)

Figure 5. Vibration response spectrum diagram under normal conditions: (a) Horizontal vibration; (b) Torsional vibration. 


\subsection{Single Misalignment Fault}

Figure 6 is the frequency domain diagram of the radial and torsional vibration of the generator rotor when the parallel misalignment is $1 \mathrm{~mm}, 2 \mathrm{~mm}, 3 \mathrm{~mm}, 4 \mathrm{~mm}$ and $5 \mathrm{~mm}$. It can be seen from the figure that the rotor response changes significantly after misalignment failure, the 2 harmonic frequency components in radial vibration and the 1 harmonic frequency component in torsional vibration appear. With the increase of misalignment faults, the radial vibration of the rotor is mainly composed of 1 and 2 harmonic frequencies. The amplitude of 1 harmonic frequency has little change, and the 2 harmonic frequency gradually increase, which is consistent with the analysis of misalignment fault excitation. The characteristic frequency is twice the frequency of the shaft, and its amplitude increases with the growth of misalignment, which is a typical feature of misalignment faults. The torsional vibration has a 1 harmonic frequency component in addition to the zero frequency component, and it also increases with the degree of misalignment faults, which is mainly affected by the bending and torsion coupling effect of the rotor as well as the parallel misalignment fault.

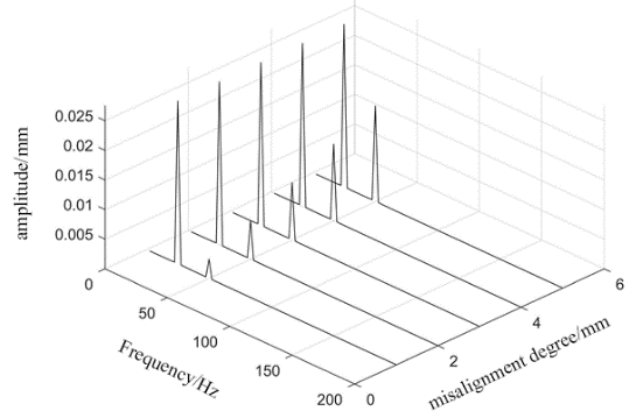

(a)

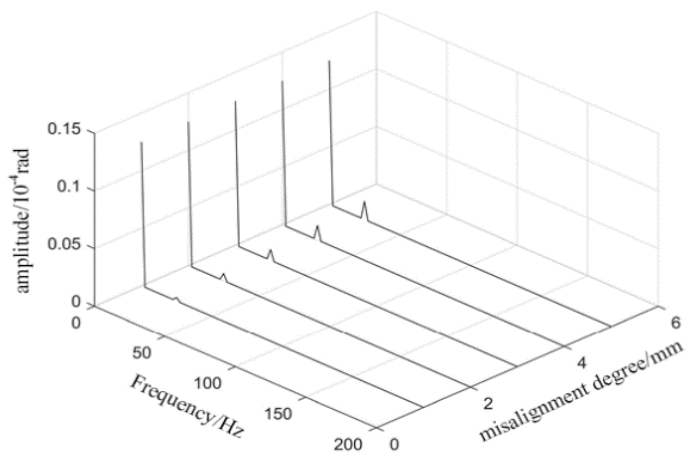

(b)

Figure 6. Vibration response frequency waterfall diagram with misalignment fault: (a) Radial vibration; (b) Torsional vibration.

\subsection{Single Short-Circuit Fault}

Figure 7 is the frequency waterfall diagram of the generator rotor system in case of $2 \%, 4 \%, 6 \%, 8 \%$, and $10 \%$ stator short-circuit fault. With the increase of the stator short-circuit fault degree, in addition to 1 harmonic frequency in the radial vibration frequency, the 3 and 5 harmonic components are constantly highlighted, the amplitude of each frequency is also increasing. The 1 harmonic is the main component of the radial vibration frequency. The 2 harmonic frequency component appears in the torsional vibration, and it increases linearly with the growth of the short-circuit coefficient. This is because the short-circuit current in the stator will induce a 2 harmonic frequency excitation current in the rotor after a short-circuit fault occurs, and then a high frequency reaction magnetic force is induced in the stator and rotor winding, thereby generating high-frequency electromagnetic force and electromagnetic torque. This is consistent with the analysis of the unbalanced electromagnetic pull force and electromagnetic torque of the generator under the short-circuit fault of the stator.

\subsection{Misalignment and Stator Short-Circuit Coupling Fault}

The generator vibration response is more complicated with the misalignment stator short-circuit coupling fault. In order to further reveal the coupling fault mechanism, this paper analyzes the vibration response characteristics of generator stator under the condition that the stator short-circuit degree and the misalignment degree are different. 


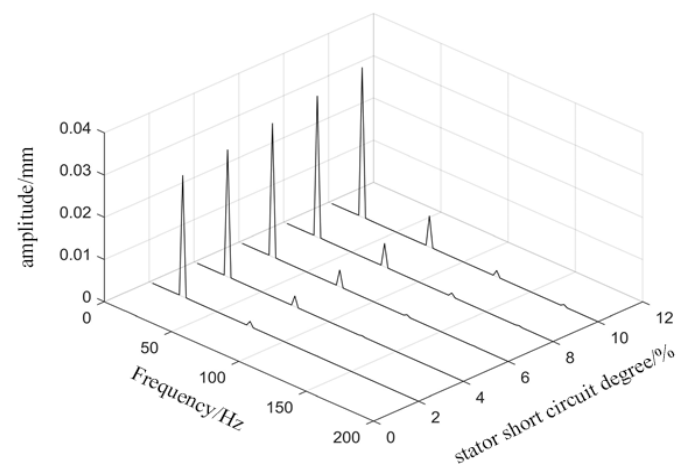

(a)

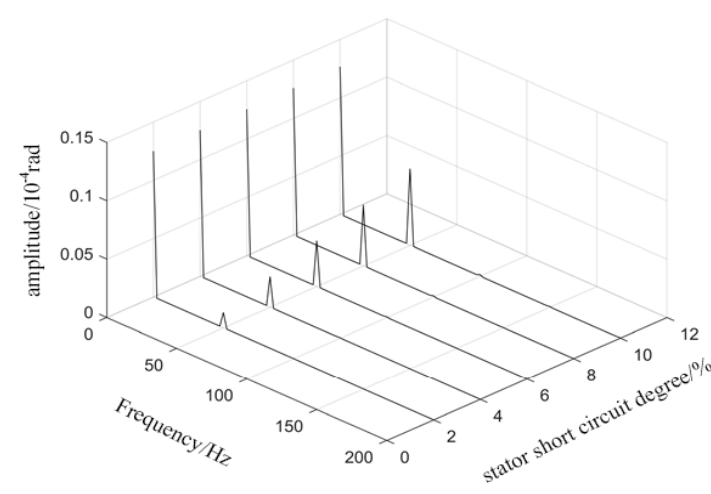

(b)

Figure 7. Vibration response frequency waterfall diagram with stator short-circuit fault: (a) Radial vibration; (b) Torsional vibration.

\subsubsection{Certain Degree of Misalignment and Varying Short-Circuit}

It can be seen from Figures 8 and 9 that with the deepening of the stator short-circuit, the time domain waveform of radial vibration of generator rotor is distorted, the peak of wave is burred, and the vibration amplitude is increased. The deformation degree of the axis track is also gradually increased, from regular half-moon shape to irregular shape.

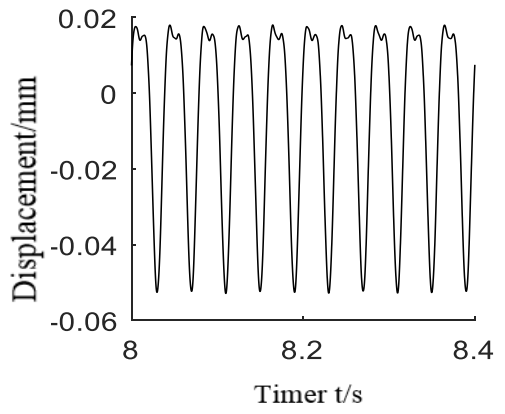

(a)

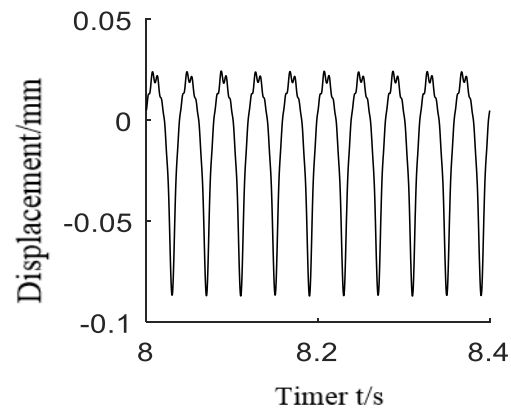

(b)

Figure 8. Time domain waveform of generator rotor vibration: (a) misalignment $3 \mathrm{~mm}$, short-circuit $1 \%$; (b) misalignment $3 \mathrm{~mm}$, short-circuit $10 \%$.

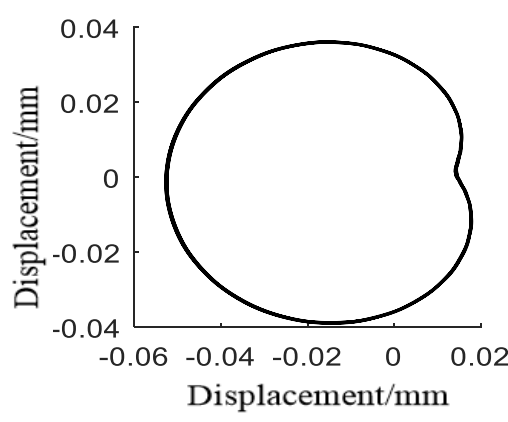

(a)

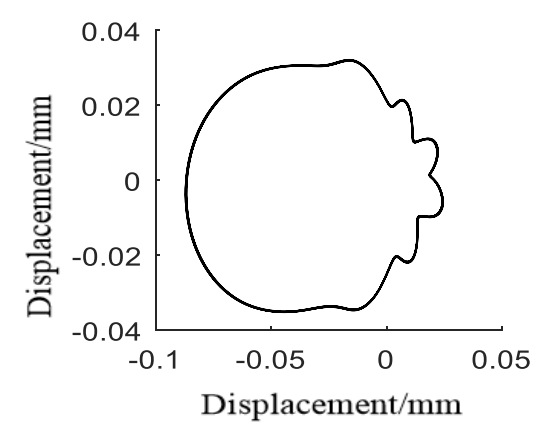

(b)

Figure 9. Vibration response axis locus of generator rotor: (a) misalignment $3 \mathrm{~mm}$, short-circuit $1 \%$; (b) misalignment $3 \mathrm{~mm}$, short-circuit $10 \%$.

As shown in Figures 10 and 11, when the amount of misalignment is fixed, with the increase of stator short-circuit degree, the 1.0 and 3.0 harmonic components of the radial vibration signal increase 
obviously, the 4.0 and 6.0 harmonic components gradually highlight, the 2.0 harmonic components of the torsional vibration signal increases sharply, and the 3.0 and 5.0 harmonic components gradually appear.

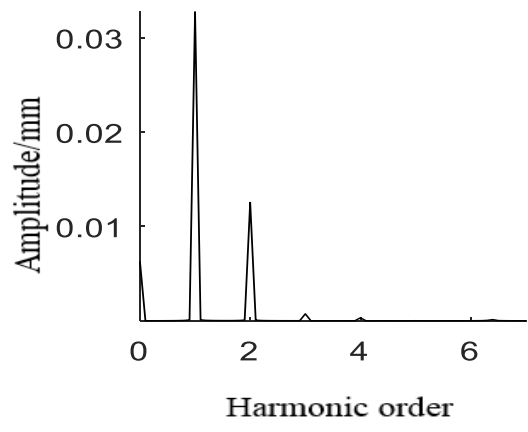

(a)

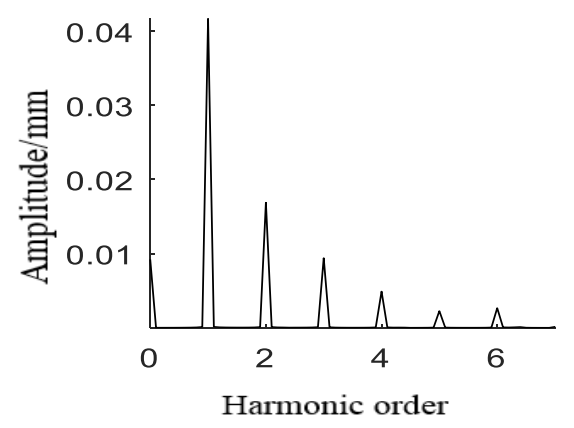

(b)

Figure 10. Radial vibration spectrum of generator rotor: (a) misalignment $3 \mathrm{~mm}$, short-circuit 1\%; (b) misalignment $3 \mathrm{~mm}$, short-circuit $10 \%$.

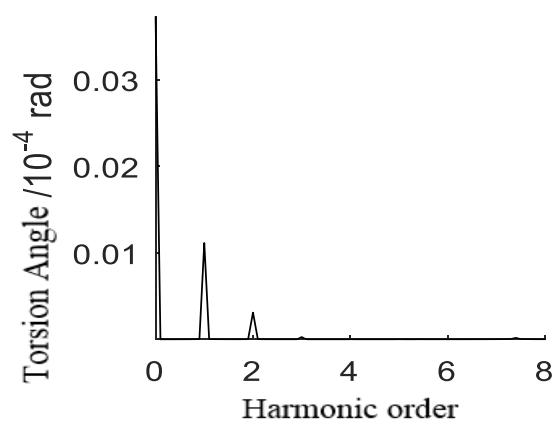

(a)

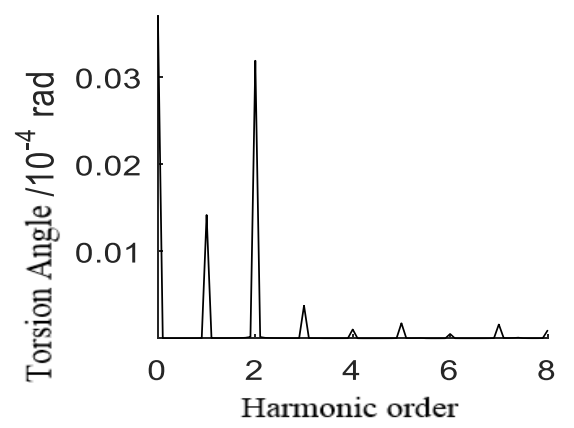

(b)

Figure 11. Torsional vibration spectrum of generator rotor: (a) misalignment $3 \mathrm{~mm}$, short-circuit $1 \%$; (b) misalignment $3 \mathrm{~mm}$, short-circuit $10 \%$.

\subsubsection{Certain Degree of Short-Circuit and Varying Misalignment}

It can be seen from Figures 12 and 13 that the radial vibration of the generator rotor increases, the time domain waveform changes from sinusoidal waveform to distorted waveform, the wave peak appears as a double wave peak phenomenon, the axis track also changes from circular shape to internal ' 8 ' shape, and the misalignment fault feature is gradually obvious.

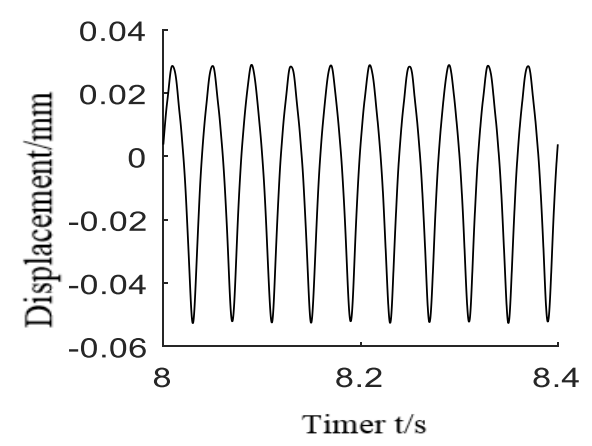

(a)

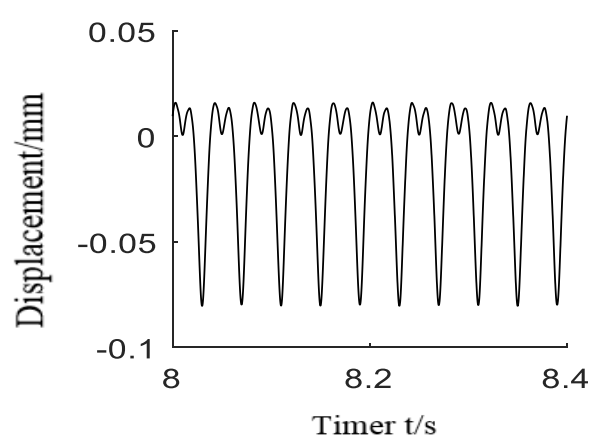

(b)

Figure 12. Vibration response axis locus of generator rotor: (a) misalignment $1.5 \mathrm{~mm}$, short-circuit $5 \%$; (b) misalignment $5 \mathrm{~mm}$, short-circuit $5 \%$. 


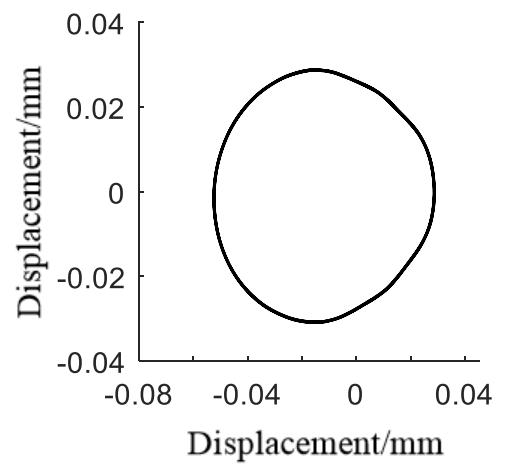

(a)

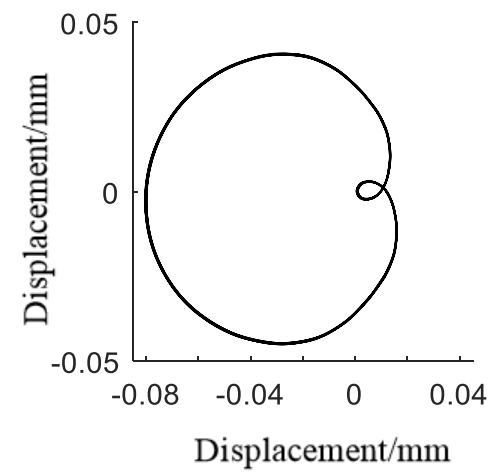

(b)

Figure 13. Vibration response axis locus of generator rotor: (a) misalignment $1.5 \mathrm{~mm}$, short-circuit 5\%; (b) misalignment $5 \mathrm{~mm}$, short-circuit $5 \%$.

As shown in Figures 14 and 15, when the amount of the stator short-circuit is fixed, with the increase of misalignment degree, the 2.0 harmonic component of the radial vibration signal increases obviously, the 4.0 and 6.0 harmonic components gradually highlight, the 1.0 harmonic component of the torsional vibration signal increases sharply, and the 3.0 and 5.0 harmonic components gradually appear.

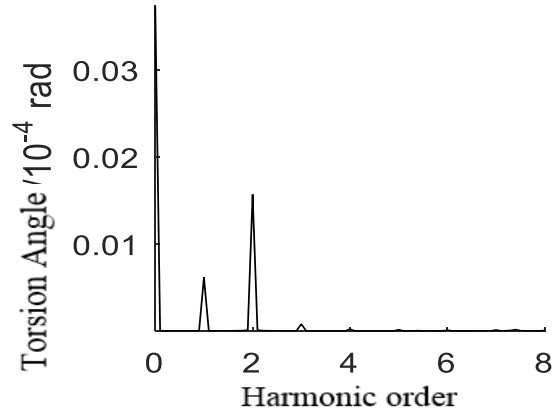

(a)

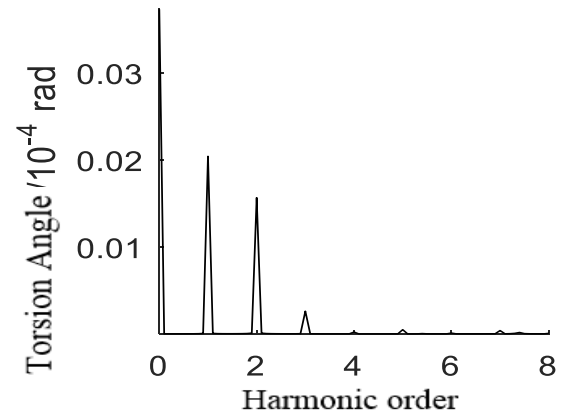

(b)

Figure 14. Radial vibration spectrum of generator rotor: (a) misalignment $1.5 \mathrm{~mm}$, short-circuit $5 \%$; (b) misalignment $5 \mathrm{~mm}$, short-circuit $5 \%$.

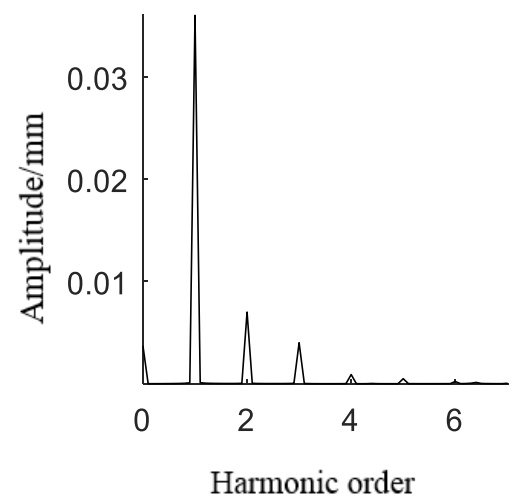

(a)

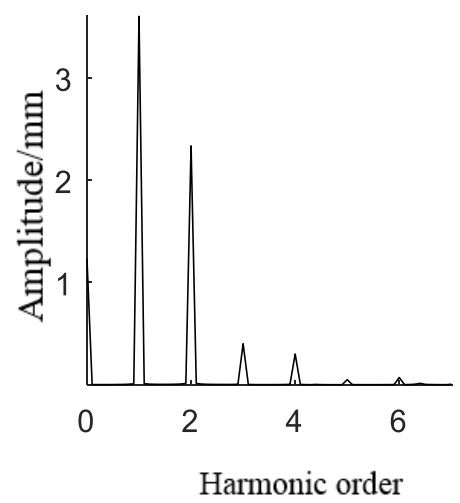

(b)

Figure 15. Torsional vibration spectrum of generator rotor:(a) misalignment $1.5 \mathrm{~mm}$, short-circuit $5 \%$; (b) misalignment $5 \mathrm{~mm}$, short-circuit $5 \%$.

The frequency waterfall diagram of radial vibration and torsional vibration response under different fault conditions are shown in Figure 16. The vibration response characteristics of the generator under the misalignment stator short-circuit coupling fault are not simply the superposition of two 
single fault characteristics, but rather, the interaction. In addition to the 1.0 harmonic of normal working conditions, the 2.0 harmonic component of misalignment, and the 3.0 harmonic and 5.0 harmonic components of stator short-circuit, the amplitude of even harmonic components in the radial vibration spectrum increases significantly with the 4.0 and 6.0 harmonic components, and the amplitude of odd harmonic components in the torsional vibration spectrum increases obviously with the 3.0 and 5.0 harmonic components.

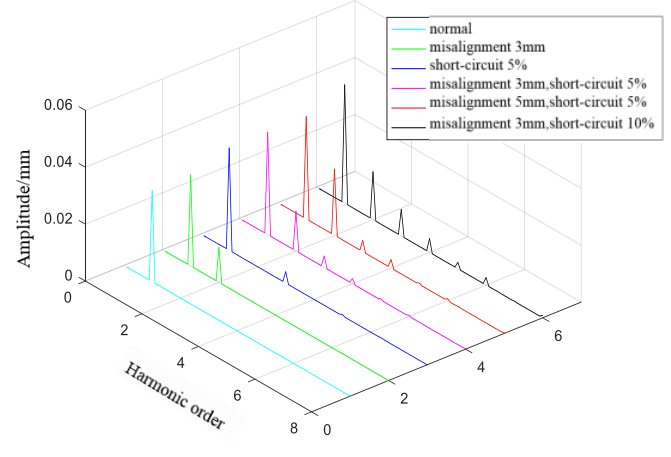

(a)

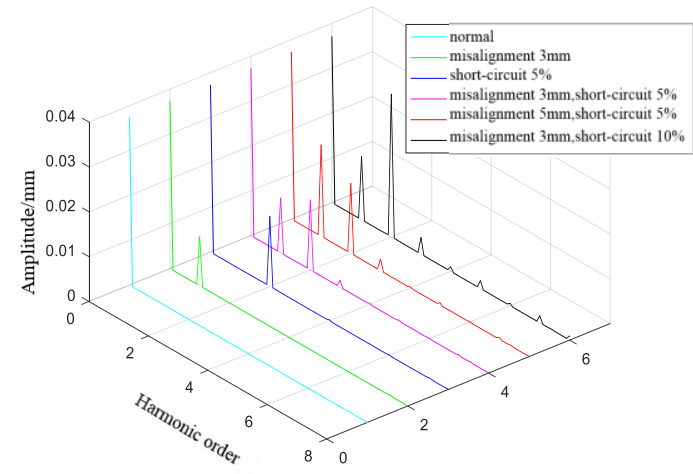

(b)

Figure 16. Vibration response frequency waterfall diagram with coupling fault: (a) Radial vibration; (b) Torsional vibration.

In conclusion, the misalignment and short-circuit coupling fault not only has a single fault characteristic, but also stimulates the high harmonic components vibration of the system, and the vibration intensity of the system is greater than any single fault of the same degree.

\section{Finite Element Analysis}

Due to the limitation of experimental conditions, the coupling fault of misalignment and stator short-circuit experiment is potentially destructive and dangerous. Therefore, the results of finite element analysis are used to verify the conclusions of numerical analysis.

According to the theory of multi-body dynamics and finite element analysis, the three-dimensional solid model is shown in Figure 17, which mainly consists of the crankshaft, piston, connecting rod, rotor, stator, bearing, coupling, flywheel and accessory components. Because the crankshaft is complex in force and bears large loads, rigid body crankshafts struggle to meet the requirement of accuracy. Therefore, the finite element analysis of the crankshaft was carried out. The rigid flexible coupling multi-body dynamic model is shown in Figure 18. Since the components are in accordance with the actual size and the material properties and density are defined, the reciprocating inertia force does not need to be considered during operation. Then, the single fault and coupling fault of misalignment and stator short-circuit are simulated by the model.

Figure 19 shows the generator rotor vibration frequency when the misalignment is constant at $3 \mathrm{~mm}$ and the stator short-circuit at $2 \%, 4 \%, 6 \%, 8 \%$, and $10 \%$ degree respectively. It can be seen from the figure that with the increase of the degree of stator short-circuit, in addition to the 1 harmonic frequency under normal conditions in the radial vibration of the rotor, the 1 harmonic frequency in misalignment condition, and the 3,4,5 and 6 harmonic frequencies in the stator short-circuit are gradually prominent. The amplitude of each frequency increases with the growth of the short-circuit degree. Among them, the increases of the 1 and 3 harmonic components are obvious. In the torsional vibration, except for the zero frequency at normal conditions, the 1 harmonic frequency during misalignment, and the 2 harmonic component when the stator is short-circuited, the 3 harmonic component gradually appears, and the amplitudes of the 2 harmonic and 3 harmonic components gradually increase with the growth 
of the short-circuit degree. The 2 harmonic component changes greatly, and the 1 harmonic component changes little.

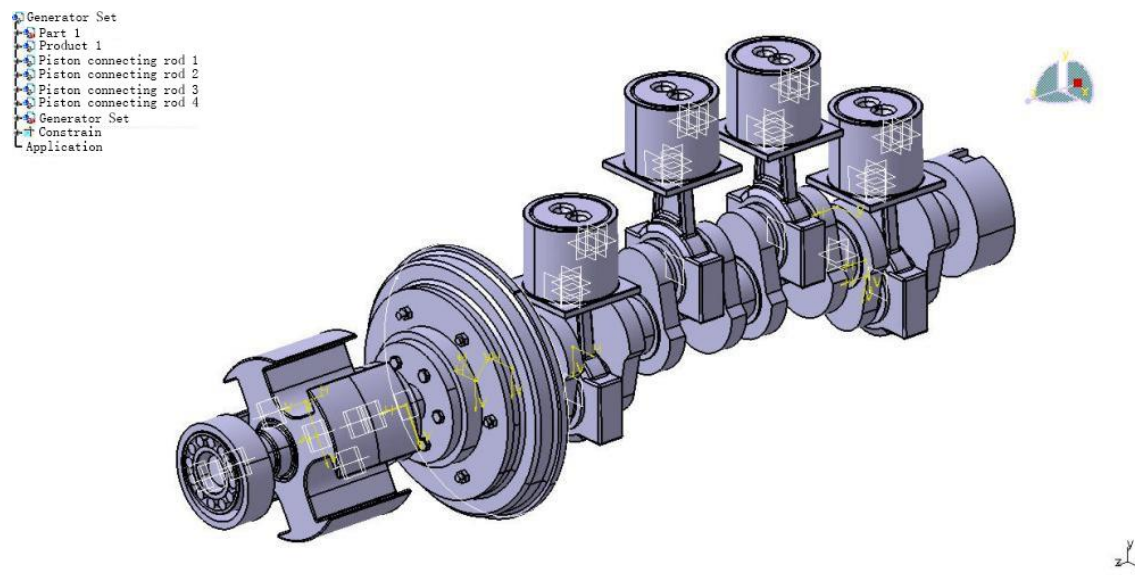

Figure 17. Three-dimensional solid model of range extender.

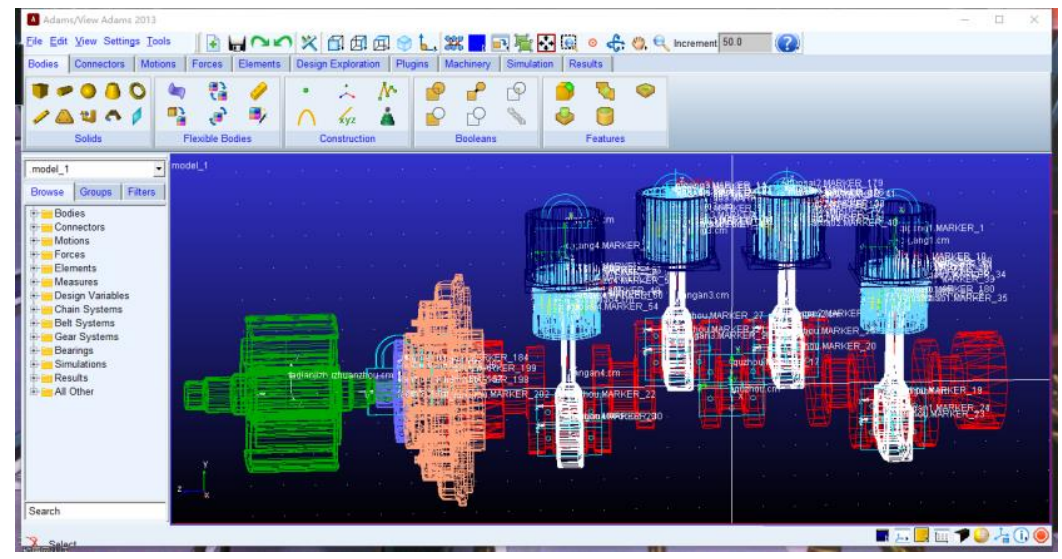

Figure 18. Rigid flexible coupling multi-body dynamic model of range extender.

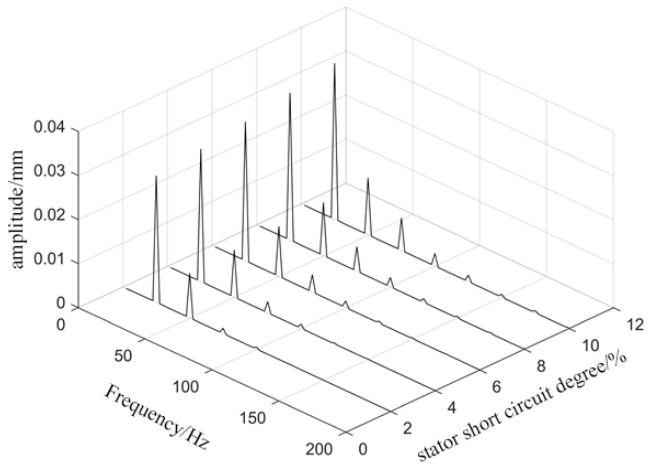

(a)

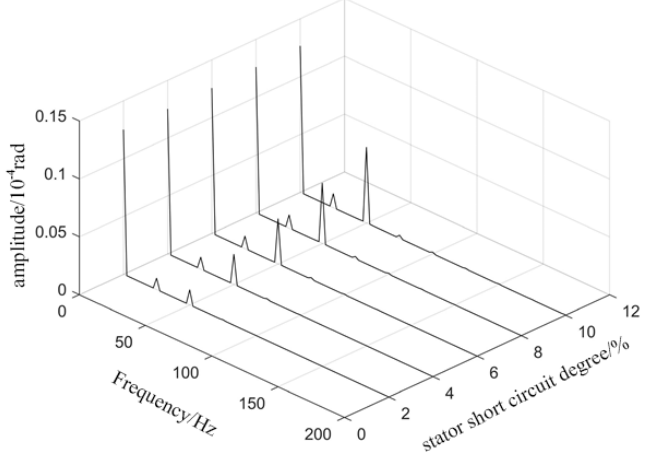

(b)

Figure 19. Coupled fault system response with misaligned $3 \mathrm{~mm}$ : (a) Radial vibration spectrum waterfall diagram; (b) Torsional vibration spectrum waterfall diagram.

Figure 20 is a waterfall diagram of the generator rotor vibration response frequency when the stator short-circuit is $10 \%$ constant and the misalignment amount is $1 \mathrm{~mm}, 2 \mathrm{~mm}, 3 \mathrm{~mm}, 4 \mathrm{~mm}$, and $5 \mathrm{~mm}$ respectively. From the figure, we can know the frequency components of the generator 
rotor radial and torsional vibration are similar to the variations of stator short-circuit degree when the misalignment amount is certain. With the increase of the amount of misalignment, in addition to the single fault frequency component in the radial vibration of the generator, there will also be the 4 and 6 harmonic frequencies, and the amplitudes are small. What is more, the 2 harmonic frequency component increases obviously with the degree of misalignment increase. The 3 harmonic component gradually appears in torsional vibration in addition to the frequency under a single fault, and the 1 harmonic component in each frequency increases significantly.

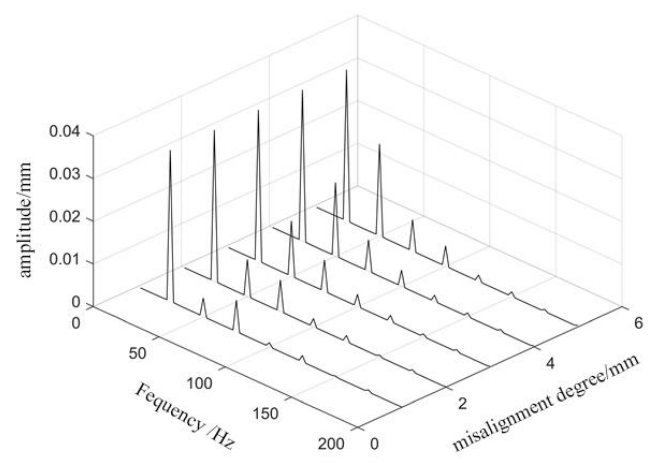

(a)

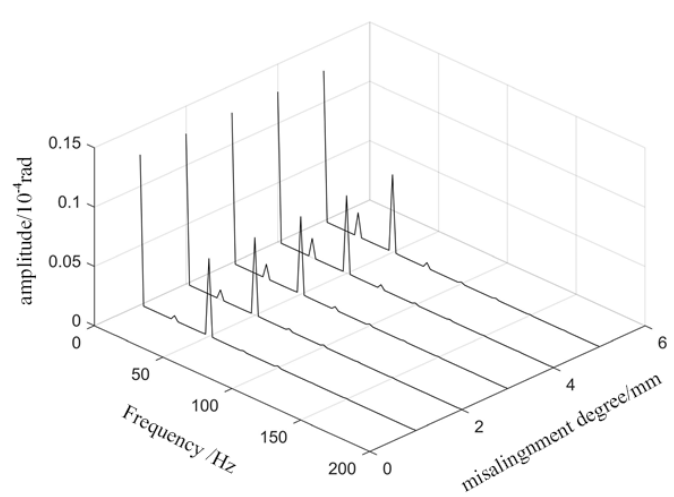

(b)

Figure 20. Coupled fault system vibration response with Stator short-circuit 10\%: (a) Radial vibration spectrum waterfall diagram; (b) Torsional vibration spectrum waterfall diagram.

Analyzing the vibration response frequency of the generator rotor under the above two coupling fault forms, it can be seen that the vibration response characteristics of the system under the coupling fault are not a set of two single fault characteristics, but rather that they interact with each other. In addition to the 1,2,3, and 5 frequencies, there are the 4 and 6 of frequency components in the radial vibration spectrum, and compared with the single misalignment fault and single stator short circuit fault, the frequency amplitude increases with the deepening of the coupling failure degree, and the change of even multiple frequency components is more obvious. In the torsional vibration, there are not only the 0,1 , and 2 harmonic frequencies, but also the 3 harmonic frequency. Compared with the single fault of the same degree, the amplitude of each high frequency component of torsional vibration increases too, and the increase of the odd multiple frequency component is more significant.

Based on the above analysis, it can be seen that the misalignment fault and the stator short-circuit fault affect each other. The coupling fault not only has the characteristics of a single fault, but also stimulates the high frequency vibration of the system, and the vibration intensity of the system is greater than any single fault of the same degree. For a single fault, the misalignment fault mainly affects the 2 harmonic frequency of radial vibration and the 1 harmonic frequency of torsional vibration, while the stator short-circuit fault mainly affects the 3 frequency, 5 harmonic frequency of radial vibration and 2 harmonic frequency of torsional vibration. At the coupling fault, the high frequency vibration of the generator rotor is excited, and the 4 and 6 harmonic frequencies of radial vibration, and the 3 harmonic frequency of torsional vibration are enhanced.

\section{Conclusions}

To reveal the misalignment and stator short-circuit coupling fault mechanism of the electric vehicle range extender, and to research the dynamic characteristics of the generator rotor under the coupling fault, this paper analyzes the excitation mechanism of the misalignment and deduces the bending and torsional electromagnetic stiffness of the generator stator short-circuit, so as to establish the mechanical model of the range extender. The torsional vibration and radial vibration response characteristics of the coupled fault system are analyzed by time domain diagram and frequency spectrum diagram. 
Using numerical analysis, this paper studies the vibration response characteristics of the generator rotor under the conditions of single misalignment fault, single stator short-circuit, and coupling fault. The results show that:

1. Single misalignment faults mainly affect the generator rotor 2 harmonic frequency of radial vibration and 1 harmonic frequency of torsional vibration; the single stator short-circuit fault mainly affects the generator rotor 3 and 5 harmonic frequencies of radial vibration and the 2 harmonic frequency of torsional vibration. Furthermore, when the misalignment fault is serious, the axis orbit of the rotor is in the shape of " 8 ", which can be used as the feature of generator misalignment fault.

2. In the vibration response of generator rotor under the coupling fault of misalignment stator short-circuit, there are not only single fault characteristic frequencies, but also high frequency multiplication components, that is to say, the even frequency doubling components of radial vibration and odd frequency doubling components of torsional vibration gradually increase, and the vibration intensity of the system is also greater than any single fault of the same degree. The 4 and 6 harmonic frequencies of radial vibration and 3 harmonic frequency of torsional vibration will gradually increase with the deepening of the coupling fault, that is to say, the coupling fault excites the high frequency multiplication vibration of the generator rotor.

Author Contributions: Conceptualization, X.X. and H.W.; methodology, J.F. and H.W.; software, J.F.; validation, N.Z.; formal analysis, X.X.; investigation, J.F.; resources, H.W.; data curation, J.F.; writing-original draft preparation, J.F.; writing-review and editing, X.W.; supervision, X.X.; project administration, X.X.; funding acquisition, X.X. All authors have read and agreed to the published version of the manuscript.

Funding: This research was funded by the National Natural Science Foundation of China under Grant 51975426 and 51505345, the Wuhan Science and Technology Project under Grant 2019010701011393, China.

Conflicts of Interest: The authors declare no conflict of interest.

\section{References}

1. Geng, C.; JIN, X.; Zhang, X. Simulation research on a novel control strategy for fuel cell extended-range vehicles. Int. J. Hydrog. Energy 2019, 44, 408-420. [CrossRef]

2. Hooshang, M.; Moghadam, R.A.; Alizadehnia, S. Dynamic response simulation and experiment for gamma-type Stirling engine. Renew. Energy 2016, 86, 192-205. [CrossRef]

3. Kruse, E.; Harrison, A.J. Active Vibration Control Technology for Electric Vehicles with Range Extender. ATZ Worldw. 2015, 117, 14-19. [CrossRef]

4. Fu, X.; Jia, W.; Xu, H.; Song, S. Imbalance-misalignment-rubbing coupling faults in hydraulic turbine vibration. Optik 2016, 127, 3708-3712. [CrossRef]

5. Li, Z.; Li, J.; Li, M. Nonlinear dynamics of unsymmetyical rotor-bearing system with fault of parallel misalignment. Adv. Mech. Eng. 2018, 10, 1-17.

6. He, Y.L.; Wang, F.L.; Tang, J.G. Effect of stator inter-turn short-circuit position on electromagnetic torque of generator with consideration of air-gap eccentricity. Trans. China Electrotech. Soc. 2017, 32, 11-19.

7. Li, J.Q.; Li, Z.H.; Tong, Z.Y. Fault diagnosis of stator inter-turn short-circuit in doubly fed induction generator based on information fusion. Electr. Mach. Control Appl. 2017, 44, 99-103.

8. Wang, S.; Sun, Y.; Huang, Z.; Mu, S. Analysis of Stator Internal Phase-to-Phase Short-Circuit in the 12-Phase Synchronous Generator with Rectifier-Load System. IEEE Trans. Energy Convers. 2018, 33, 299-311. [CrossRef]

9. Bouzid, M.B.K.; Champenois, G.; Tnani, S. Reliable stator fault detection based on the induction motor negative sequence current compensation. Int. J. Electr. Power Energy Syst. 2018, 95, 490-498. [CrossRef]

10. Berzoy, A.; Mohammed, O.A.; Restrepo, J.A. Analysis of the Impact of Stator Interturn Short-Circuit Faults on Induction Machines Driven by Direct Torque Control. IEEE Trans. Energy Convers. 2018, 33, 1463-1474. [CrossRef]

11. Yuan, J.F. Fault Analysis and Diagnosis of Inter-Turn Short-Circuit of Large Turbogenerator; North China Electric Power University: Beijing, China, 2017. 
12. Sawalhi, N.; Ganeriwala, S.; Tóth, M. Parallel misalignment modeling and coupling bending stiffness measurement of a rotor-bearing system. Appl. Acoust. 2019, 144, 124-141. [CrossRef]

13. Taplak, H.; Erkaya, S.; Uzmay, İ. Experimental analysis on fault detection for a direct coupled rotor-bearing system. Measurement 2013, 46, 336-344. [CrossRef]

14. Solmaz, H.; Karabulut, H. A mathematical model to investigate the effects of misfire and cyclic variations on crankshaft speed fluctuations in internal combustion engines. J. Mech. Sci. Technol. 2015, 29, 1493-1500. [CrossRef]

15. Liao, M.F.; Liang, Y.Y.; Wang, S.J.; Wang, Y.K. Misalignment in drive train of wind turbines. Mech. Sci. Technol. Aerosp. Eng. 2011, 30, 173-180.

16. Li, M.; Li, Z.G.; Zhang, Y.B. Non-linear dynamics of unsymmetrical generator rotor systems with parallel misalignment. Proc. Chin Soc. Electr. Eng. 2012, 32, 111-118.

17. Wang, L. Analysis and Diagnosis of Vibration Characteristics of Rotor Misalignment in Turbogenerator; North China Electric Power University: Beijing, China, 2011.

18. Wu, W.J. Analysis and Research on Vibration Characteristics of Generator Stator Winding in Turn to Turn Short-Circuit; North China Electric Power University: Baoding, China, 2010.

19. He, Y.L.; Peng, B.; Wan, S.T. Effect of stator interturn short-circuit position on stator vibration of generator. J. Vibration Eng. 2017, 30, 679-687.

20. Ma, M.K. Analysis and Diagnosis of Composite Characteristics of Inter-Turn Short-Circuit Faults in the Field Winding of Turbo-Generator; North China Electric Power University: Beijing, China, 2019.

21. Liu, Z.X. Mechanism Analysis and Feature Extraction of Coupling Fault of Range Extender in Electric Vehicles; Wuhan University of Science and Technology: Wuhan, China, 2019.

22. Xu, X.; Wang, H.; Zhang, N.; Liu, Z.; Wang, X. Review of the fault mechanism and diagnostic techniques for the range extender hybrid electric vehicle. IEEE Access 2017, 5, 14234-14244. [CrossRef]

23. Wang, N.; Jiang, D. Vibration response characteristics of a dual-rotor with unbalance-misalignment coupling faults: Theoretical analysis and experimental study. Mech. Mach. Theory 2018, 125, 207-219. [CrossRef]

24. Wang, F.L. Torque Characteristic Analysis of Compound Fault of Generator Air Gap Eccentricity and Stator Short Circuir; North China Electric Power University: Beijing, China, 2016.

(C) 2020 by the authors. Licensee MDPI, Basel, Switzerland. This article is an open access article distributed under the terms and conditions of the Creative Commons Attribution (CC BY) license (http://creativecommons.org/licenses/by/4.0/). 